\title{
Using Activity Theory to Conceptualize Online Community and Using Online Community to Conceptualize Activity Theory
}

\author{
Sasha Barab, Steve Schatz, and Rebecca Scheckler \\ School of Education \\ Indiana University
}

\begin{abstract}
In this article we describe the evolving structure of the Inquiry Learning Forum (ILF), a sociotechnical interaction network (STIN) designed to support a Web-based community of in-service and preservice mathematics and science teachers sharing, improving, and creating inquiry-based pedagogical practices. Specifically, we apply activity theory as an analytical lens for characterizing the process of designing and supporting the implementation of this online community. Our findings lend support for three implications. First, activity theory can provide a useful analytical tool for characterizing design activity, especially in terms of illuminating the challenges of designing something like community. Second, as one moves toward trying to design a community, particularly one in which the members will be expected to engage in new practices that challenge their current culture, many tensions emerge. Third, consideration of the ILF as a STIN was a necessary conceptual step in our understanding of the ILF and the transactional nature of people and tools. It is our conception that activity theory and STIN are synergistic theoretical frameworks that, when taken together, can provide a richer view of design activity and community functioning than either can offer in isolation.
\end{abstract}

\section{INTRODUCTION}

It is common for researchers of educational technologies to publish case studies that report the character of systems they develop in terms of a unitary, coherent, and refined entity. Although these end-product characterizations provide a useful exemplar, they fail to portray (and acknowledge) the complex dynamics that are characteristic of the making and use of these systems. In practice, the development and use of new educational technologies are much more fragile and uncertain than is captured by portrayals that black-box the evolving dynamics that bring about technological developments and characterize their implementations (Latour, 1987). Although these complexities are evident in the production of technical artifacts such as a microchip or a computer keyboard, they are even more pronounced when building electronic spaces such as online communities in which users are expected to virtually visit and interact with other users (Kim, 2000; Preece, 2000; Smith \& Kollok, 1999). This pronounced effect is evident in part because the use of electronic col-

Requests for reprints should be sent to Sasha Barab, School of Education, Indiana University, Room 2232, 201 North Rose Avenue, Bloomington, IN 47405. E-mail: sbarab@indiana.edu 
laborative spaces is not simply defined by their technical structure but may be better described as sociotechnical interaction networks (STINs), the latter term highlighting the social relationships through which these technical structures take on meaning (Kling, McKim, Fortuna, \& King, 2001, personal communication). In this article, we use both activity theory, specifically Engeström's widely used framework for applying activity theory, and STINs to describe the Inquiry Learning Forum (ILF), a large Web-based research and development project.

The concept of STIN captures our belief that technologies are developed within and can influence and be influenced by the dynamics of the social world. According to Kling et al. (2001), a STIN includes people (including organizations), data, equipment, documents and messages, legal arrangements and enforcement mechanisms, and resource flows. From a STIN perspective, any characterization of the technology (e.g., an online community) must capture the networks of interactions among people that both define and are defined by the technology. The ILF, an example that includes an electronic Web site (the e-ILF) and its associated networks of people and places and other tools, is designed to support a Web-based community of inservice and preservice mathematics and science teachers sharing, improving, and creating inquiry-based pedagogical practices (http://ilf.crlt.indiana.edu). The hallmark of this environment is that teachers with a broad range of experience and expertise come together in a virtual space to observe, discuss, and reflect on pedagogical theory and practice anchored to actual teaching vignettes. The ILF design centers around the vision of a community in which teachers can virtually visit each other's classrooms to observe and discuss approaches to teaching mathematics and science topics and to share artifacts. By looking at the ILF as a STIN, we see that it is a broad activity system and not simply a ready-made technical structure.

Designing online spaces for collaboration (i.e., building for the ILF) is a complex activity that can be difficult to characterize and describe to others. Additionally, when developers embrace a participatory design commitment (Schuler \& Namioka, 1993) and try to build something like community ${ }^{1}$ (Barab \& Duffy, 2000), the disparity between initial design conceptions and what actually occurs in practice can be great. This disparity occurs because if one truly wants to design with the users, as should be the case if one wishes to support the emergence of community, then the product itself must grow from within, based on the contextualized needs and constraints of the users. However, characterizing the design and implementation of these types of sociotechnical, large-scale projects in their contextualized splendor is not a straightforward task. It requires representing a dynamic and evolving system in a way that preserves these dynamics without minimizing the complexity of the system. We, and others (Nardi, 1996), have found activity theory to

\footnotetext{
${ }^{1}$ Based on a review of the literature and our previous work we define online community as " a persistent, sustained social network of individuals who share and develop an overlapping knowledge base, set of beliefs, values, history, and experiences focused on a common practice and/or mutual enterprise" (Barab, MaKinster, \& Scheckler, 2003, p. 238). Communities, according to Barab et al. (in press), have (a) shared knowledge, values, and beliefs; (b) overlapping histories among members; (c) mutual interdependence; (d) mechanisms for reproduction; (e) a common practice and/or mutual enterprise; (f) opportunities for interactions and participation; (g) meaningful relationships among members; and (h) respect for diverse perspectives and minority views. As such, although the use of the term community in referring to the ILF members and their transactions may be a little presumptuous in terms of the evolutionary time frame of this data collection (only 2 years into the project), the term captures the underlying commitments of the researcher-designers. Further, at the time of this revision (30 months since project inception), the ILF has over 1,400 members who, collectively, logged over 800 posts and accessed the Web site over 20,000 times in the month of December 2001.
} 
provide a useful analytical lens for characterizing and representing these dynamics while preserving their complexity in vivo.

In particular, we use Engeström's $(1987,1993,1994)$ framework to characterize the ILF and to examine how its development and implementation differed from our initial conceptions at the outset of the project. In Engeström's (1987) framework, all activity is treated as part of a rich dynamic in which the relations among the actors (participants) and the objects they act on are mediated by tools, rules, communities, and divisions of labor. At the same time as we demonstrate the usefulness of activity theory as an analytical lens, we also demonstrate the usefulness of conceptualizing the online community as a STIN. These two frameworks, activity theory and STIN, are synergistic and when taken together can provide a richer view of the design activity and community functioning than either can offer in isolation.

\section{CHARACTERIZING THE ILF AS A SYSTEM}

As described here, in telling the story of the making and implementation of the ILF, we found it useful to ground our explanations in terms of both activity theory and STIN frameworks. Whereas the STIN framework acknowledges technologies as networks of transacting social and technical components, activity theory provides an analytical lens and framework for examining activity where "the analyst constructs the activity system as if looking at it from above" (Engeström, 1999, p. 10). In this characterization we view theory, such as activity theory and STIN, as an entry point to analysis and understanding and not as truth. In an important sense, then, theory making becomes another type of practice.

\section{Activity Theory}

In conceptualizing the making of the ILF, we draw on the theoretical perspective of activity theory (Engeström, 1987, 1993, 1999; Leont'ev, 1974, 1981, 1989), referring to a line of theorizing that was initiated by Leont'ev, Vygotsky, Luria, and Marx. Activity theory provides a theoretical framework for examining different forms of human praxis (Kuutti, 1996), including learning (Barab, Barnett, Yamagata-Lynch, Squire, \& Keating, 2002), human-computer interactions (Nardi, 1996), doctor-patient relations (Engeström, 1993), instructional designs (Jonassen \& Rhorer-Murphy, 1999), and knowledge-building communities more generally (Engeström, 1999). Given its emphasis on the reciprocal nature of learning and doing, of tool use and community, and of content and context, activity theory has much to offer in tackling the theoretical and methodological questions that are central when one conceives of a technology as a STIN.

Activity theorists are not simply concerned with doing as disembodied action but are referring to doing to transform some object, with a focus on the contextualized activity of the system as a whole (Engeström, 1987, 1993; Kuutti, 1996). As conceptualized by Engeström (1987), an activity system is made up of a subject (individuals or groups that act and whose agency is selected as the point of view for the analysis) and an object (that which is acted upon), as well as the components (tools [conceptual and physical], community, rules, and divisions of labor) that mediate the relations of subject and object. Activity systems are also constrained by the formal (systematic, 
general, and expected), informal (idiosyncratic adaptation), and technical (mandated and, potentially, written) rules, norms, and conventions of the community.

Although Engeström's well-known triangle provides explanatory utility, Garrison (2001) critiqued it and activity theory more generally in terms of compartmentalizing a transactional process. Garrison, in discussing transaction, turned to Dewey and Bentley (1949/1989) who distinguished among three forms of action:

(1) Self-action: where things are treated as functioning independently and viewed as acting under their own powers; (2) Inter-action: where one thing is balanced against another thing in casual interconnection; and (3) Trans-action: where systems of description and naming are used to deal with aspects and phases of action, without attribution to "elements" or other presumptively detachable or independent "entities," "essences," or "realities," and without isolation of presumptively detachable "relations" from such detachable "elements." (pp. 101-102)

A key concept in transaction is the interdependency and interconnection of components that only remain separate in name or in researchers' minds, for in their materiality they change continuously in relation to other components. The notion of an ecological system is a good example of many transacting elements that can be understood better as a dynamic system than as a group of static individuals. Garrison (2001) argued that activity theory treats system components as interacting as opposed to transacting. Although the former characterization treats each component as part of the interaction, it does not adequately acknowledge that all components, when examined in the context of activity, are simply transactional subfunctions of a larger function-the activity. When one adopts a transactional lens in understanding activity systems, the system is treated as permeating all components with the triangle simply describing a lens through which to examine the overall system in its multiple functions. For example, a tool is not an independent entity but is instead a description of a subfunction or a particular perspective from which to understand the larger activity. In a transactional system, mediation changes its character radically from dialectical to symmetrical (Miettinen, 1997). This shift in our understanding of the ILF as a structure, where human and technological actors transact, gave us useful insights into the functioning of this system. We return to this argument later when we characterize and interpret the current ILF system. In general, we had greater design success once we made the shift from viewing the e-ILF as a tool that mediated activity to looking at the e-ILF as part of a dynamic and transacting system, the ILF. In this shift, we were aided by the concept of a STIN, and we have come to synergistically use both theories in ways that reveal richness at different levels of analysis. It is our experience that drawing on both perspectives allowed us to more usefully characterize, and thus design, the ILF than we were able to do when we constrained our thinking in terms of only one or the other theoretical perspective.

\section{Characterizing Social Transactions as a STIN}

It is important to point out that the components of activity systems are not static components existing in isolation but are instead considered as reciprocally interacting with and reciprocally constituted through interactions with the other components that constitute the activity system as a whole. As such, an examination of any phenomenon (e.g., the ILF) must consider the dynamics among all these components. In addition to these interactions of an activity system of a particular time and 
space, an activity system is made up of nested instances of activities, all of which constitute the current system. Barab, Barnett, Yamagata-Lynch, et al. (2002) described it this way:

Although the computer may serve as a tool in one activity system, at an earlier time this computer may have been an object or an outcome of another activity system or of another instance of the same activity system in which it is now a tool (Latour, 1987). In a similar fashion, technical rules that affect a current activity system (e.g., syllabus-delineated requirements) were the outcome of previous instances of activity in which the technical rules were created. (p. 23)

This perspective extends the unit of analysis from the mind of the individual (as in traditional cognitive research) or from the human-computer interaction (as in traditional Human-Computer Interaction research) to the entire activity system (Barab, Barnett, Yamagata-Lynch, et al., 2002).

The important design point here is that designers shift their focus from simply supporting usability to supporting what Preece (2000) described as "sociability." Barab, MaKinster, Moore, Cunningham, and The ILF Design Team (2001) described sociability as "those social policies and technical structures that support the community's shared purpose and social interactions among group members" (p. 83). In this focus, and especially when one moves toward online communities (Barab, MaKinster, \& Scheckler, in press; Smith \& Kollok, 1999), the tools supporting learning are not simply those that are electronic in nature but include those individuals with whom learners (community members) transact. It is for this reason that Kling et al. (2001) introduced the term sociotechnical interaction networks, referring to a framework for conceptualizing human behavior that occurs in technology-mediated social settings. ${ }^{2}$ The power of the STIN framework is its base assumption that all technical structures must be understood as part of the context of social transaction through which they are constituted and through which they take on their meanings. Researchers applying the STIN framework treat these social and technical nodes as part of a dynamic transacting system that functions as a transactive unit. Although Kling et al. derived STIN for scientific communities using multiple-media forms that are not patently educational, Kling and Courtright (in press) recently demonstrated their use for educational settings. It is drawing on this STIN framework and with a recognition of the continual transactions that humans (individuals, groups) and artifacts of various kinds (computers, electronic journals, videos, lesson plans) have in online communities that we use activity theory as an analytical lens to understand the system dynamics of the ILF community—-thus demonstrating the synergistic nature of activity theory and STIN.

\section{THIS STUDY}

The focus of this analysis is twofold: (a) the making of the ILF from a researcher/designer perspective, and (b) the using of the ILF in practice from a teacher perspective. Additionally, we are discuss-

\footnotetext{
${ }^{2}$ Others have debated the value of actor-network theory versus activity theory. Their differences notwithstanding, we view the STIN framework and its focus on transaction (as opposed to the actor-network theory focus on tracing the historical development of actors whether they are human or nonhuman) as complementary to activity theory and not antagonistic. Kling et al. (2001) modified actor-network theory to derive the concept of STIN. For our purposes, the major difference between STIN and actor-network theory is the ability to present the entire STIN in a speculative way rather than having to wait for this STIN to develop in use. In other words, although actor-network theory is particularly useful for tracing the historical development of a sociotechnological network over time, STIN is useful for observing the dynamic transactions of a system as a simultaneously functioning unit.
} 
ing the ILF as both an electronic tool (the e-ILF) and, consistent with the STIN perspective, an entire network that includes people and technical artifacts so that we are differentiating between the e-ILF (a tool) and the ILF (the e-ILF and its inseparable transactions). The people include researchers, designers, and programmers, as well as the teachers and administrators at school sites. The artifacts include meeting rooms (where ILF designs are discussed), telephones, computer networks, video cameras, automobiles, notebook computers, and numerous software applications. In carrying out our analysis, we use activity theory to characterize both the processes involved in the making of the ILF and the activity of the ILF teachers. We begin with a characterization of our conceptions of the design and then the implementation processes during the 1st year of the project. This is then contrasted with our Year 2 conceptions in which we acknowledge the ILF as a sociotechnical entity. Last, after we conceptualize the ILF as a STIN, we return to activity theory as a lens from which to understand the STIN functioning as subject, tool, object, community, and outcome.

\section{Research Framework}

Our research can best be described as naturalistic inquiry, with interpretations based on qualitative data (Guba \& Lincoln, 1983; Scriven, 1983). Some interpretations were based on ILF team members ("insiders") writing about their experiences and on their perspectives about events within which they directly participated. Other interpretations were developed by two researchers ("outsiders") who were hired to observe the ILF team as they made and researched the ILF. This research team, in addition to attending meetings and taking field notes, conducted semistructured interviews with both the member participants and the designers. The interviews consisted of more than 20 questions that covered a wide range of project design issues. The questions were derived from the conversations with project team members and analysis of literature on knowledge networks, and they continually evolved through group meetings. The interviews were carried out by two researchers and were recorded on audiotape or, in some cases, involved team members completing Web-based forms. The interviews typically lasted 60 to $90 \mathrm{~min}$.

In addition to these sources of data, interpretations were triangulated using field notes, interviews, document analysis, and member checks (Lincoln \& Guba, 1985). Some of these data were collected as the events occurred, and other data were based on retrospective recall through interviews and examinations of traces. For example, in reviewing 9 months of shared e-mail, early iterations of documents, and project notebooks, the goal was to use these multiple sources of data to build a story of the making of the ILF. Data collection efforts resulted in a large corpus of data, including field notes, interview transcripts, design artifacts at various stages in the project, project record keeping, meeting notes, e-mail interactions, ethnographic observation of the online space, and interviews with the research and design team as well as with teacher-users of the e-ILF.

We used the tenets of activity theory to inform our interpretations. In fact, much of our discussion in the results and the conclusions sections focuses on interactions and tensions among the core components of activity systems. Using activity theory as our theoretical lens and as an analytical tool, it was our intention to reflect on the emergent issues in terms of the relations of subject and object as mediated by the primary components that Engeström (1987) described as constituting an activity system. In addition to identifying the core components, we identified the pervasive tensions that characterize the described activity systems (Leont'ev, 1974; Engeström, 1987, 1993). It is also important to acknowledge our interventionist stance as design-based researchers 
who have a hand in designing the very contexts in which we are researching (Brown, 1992). Similar to the developmental work research conducted by Engeström (1994), this interventionist stance requires us to be both researcher and intervening participant-being engaged in forming new cultural artifacts and forms of practice jointly with the community members at the same time we are researching their formation.

An important part of characterizing and facilitating system dynamics is to identify tensions and understand how they contribute to system functioning and evolution. Understanding these tensions and their role in driving community life is instructive for characterizing the nature (and potentially supporting the evolution) of a system (Barab et al., 1999). Engeström (1999) viewed tensions as characterizing system activity and driving system innovation, and Wenger (1998), focusing on community, discussed the importance of understanding the interplay of system dualities (reification vs. participation; designed vs. emergent; local vs. global). These dualities refer to core struggles that are endemic to system activity and that characterize the design struggles of such a system within these dualities, characterizing system activity and driving system innovation (Barab, Barnett, \& Squire, 2002; Engeström, 1999). Wenger (1998) wrote, "a duality is a single conceptual unit that is formed by two inseparable and mutually constitutive elements whose inherent tension and complementarity give the concept richness and dynamism" (p. 66). In other words, even though the analytical lens of dualities provides a useful framework for characterizing community dynamics, its usefulness as a design/research framework lies in uncovering the system dynamics and understanding how the interplay between both sides of the duality (as well as among dualities) contributes to community life.

\section{ANALYSIS AND DISCUSSION}

The evolution of the STIN of the ILF has progressed through multiple iterations that we roughly characterize in terms of two stages: building a Web site (the e-ILF) and supporting the STIN (the ILF). Following a description of the first stage, we present the generalized activity system as viewed through Engeström's (1987) lens for both the design and envisioned teacher usage of the ILF. For the sake of discussion we create a divide between these two sets of activities, but in practice the lines between them were blurry if they existed at all. The current usage discussion includes a description of the last stage, conceptualizing these in terms of the STIN framework and then using activity theory to better understand its various ways of functioning.

\section{Stage 1: Building a Web Site (1-12 Months)}

Although the Web-based community discussed in this article emerged out of a number of influences, the primary design impetus was the funding of a 3-year research grant from the National Science Foundation (NSF). The grant's purpose was to support the design and research of a virtual community of in-service and preservice mathematics and science teachers sharing, improving, and creating inquiry based pedagogical practices-what has become the ILF. More specifically, it was funded to build an Internet space based on a "visiting the classroom" metaphor, and with an internal desire to support teachers in becoming full participants in and owners of their virtual space. Although building an electronic knowledge network was a part of the proposal, this was also a re- 
search grant. The research goal of this project was to understand the principles for fostering, sustaining, and scaling communities of practice in which the value to participants of sharing their practice and entering in the dialogue outweighs the costs of participation (e.g., time, technology access, and the concerns of letting others view one's teaching). Although the effective use of technology in supporting a community of practice is a focus of this research, it is clear that technological environments are only one component of an overall community-building strategy. Thus, this research project examines the variety of variables that impact the dynamics of the social networks through which teachers seek to improve and share their pedagogical practices.

Those familiar with the funding process know that for every grant that is funded, several grants are written. Researchers become accustomed to "casting their bread upon the waters"-roughly outlining the ideas that they would like to pursue. However, there is an enormous difference between conceptualization for the purpose of writing a grant and the reality of a funded project. In our case, although much thought, development, and testing had already been done, the first 6 months of the grant were largely focused on development of the e-ILF Web site. Although members of the community of teachers for whom the e-ILF was targeted were consulted on occasion for feedback and user testing, the Herculean task of creating a site that pulled together many existent technologies in a new and powerful way was too time-consuming to expect ongoing, active participation.

At this point (6 months into the process), we had a conception of the guiding design commitments and a rough sketch of what the e-ILF would look like. Four design commitments guided the ILF project design (Barab, MaKinster, Moore, Cunningham, \& The ILF Design Team, 2001):

1. Foster Ownership and Participation. We believe that a truly effective professional development environment must include a community of professional practitioners with varied experiences and skills who accept responsibility for building and maintaining their environment.

2. Focus on Inquiry. Our goal is to foster inquiry, both in terms of inquiry pedagogy in the classroom and teacher inquiry into his or her own practices.

3. Visit the Classroom. A central strategy in the design and implementation of our knowledge network is the use of video streaming and Web-based technologies to situate participants in the social context of other community members' teaching practice.

4. Support Communities of Purpose. We hope to bring together and support groups of teachers organized around some collective experience and curricular interest.

The majority of activity centered on the development team. Development team meetings were large and exciting. Graduate students and faculty who had never before undertaken a project of this magnitude worked together to create a powerful tool for professional development. Adding to the challenge, one of the Principal Investigators (PIs), and a central figure in the conceptualization of the grant, took a leave of absence to work with an Internet start-up just as the funding was released. Faculty who had intended to have a limited involvement in the grant stepped forward to provide leadership.

The research team developed data collection instruments. In addition, they oversaw the direction of development, ever mindful of the charge that this was also a research project. There was some consideration of how to attract users to the site (generally expressed as, "If we build it, will they come?"), but most took a wait-and-see attitude. In April 2000 (9 months into the process), version 1.0 of the e-ILF was developed and put on a password-protected Web site for all 6th-grade 

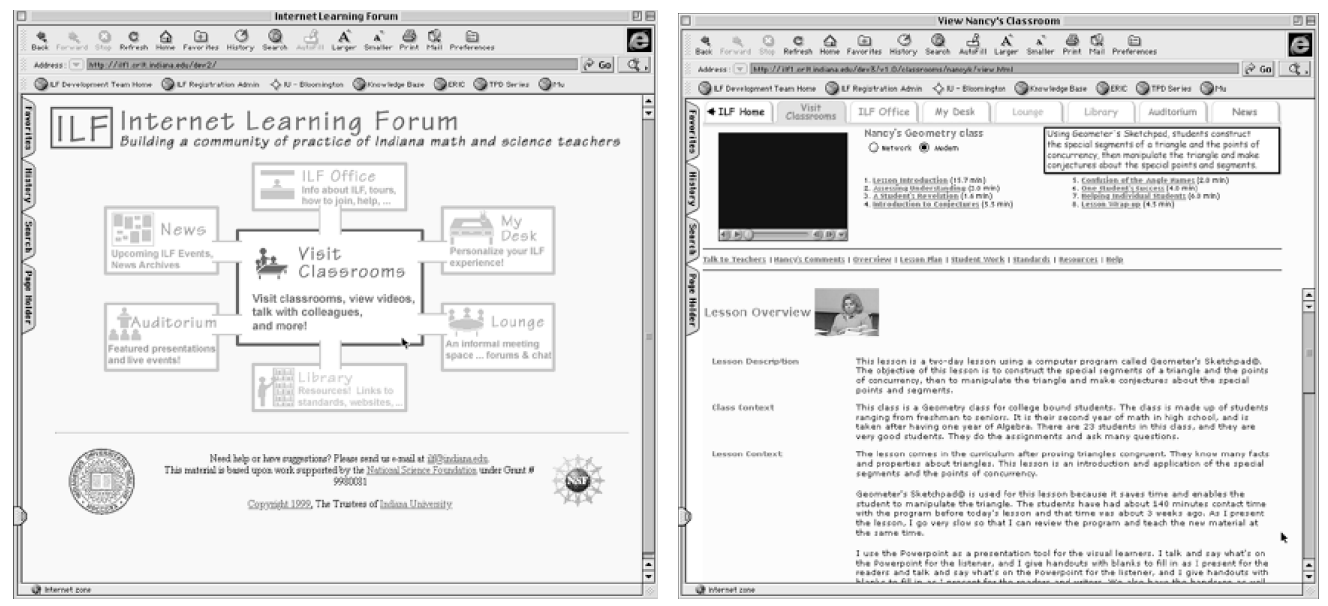

FIGURE 1 First iteration of the ILF home screen, consisting of links to the Visit Classrooms, ILF Office, My Desk, Lounge, Library, Auditorium, and News. Also included is a current screenshot of a specific Classroom, including links to an overview of the lesson, reflective commentary, descriptions of teaching activity, lesson plans, student examples and connections with both state and national standards.

through 12th-grade math and science teachers in the state of Indiana. At this point, the ILF consisted of a variety of participant structures, all related to virtually visiting the classrooms of other teachers. The home screen of the ILF is shown in Figure 1 with the Visit Classrooms location being the central space. The Visit Classrooms space was developed by working with "contributing members" to videotape a lesson they were teaching, to gather relevant materials (lesson plans, student work, relevant standards), to segment the lesson into smaller clips to be digitized for the Internet, and to gather teacher reflections on each clip.

Once the ILF members enter a classroom, they can view an overview of the lesson, reflective commentary, descriptions of teaching activity, lesson plans, student examples, and connections to both state and national standards in addition to the classroom video clips. Along with the Visit Classroom spaces, there were five other virtual spaces designed to support professional development needs: The ILF Office (offers help and other ILF information), News Room (lists current events), Lounge (hosts general conferences), Auditorium (supports video-casting live or canned presentations), Library (houses reference links and files of interest), and My Desk (stores personal bookmarks to resources, discussions, and classrooms).

Although there were many technological challenges, the site worked even when teachers logged in from home over a modem. Feasibility was not a concern. Instead, most participant concerns involved whether to let people outside the target population register to enter the password protected e-ILF. These gatekeeping considerations generally involved out-of-state teachers (the grant was specifically for Indiana teachers) or groups that wanted to participate as an independent subset within the e-ILF. During these considerations, the research team continually revisited the tension between research and service: If this is a research project, the population parameters are very important; if this is a service project, we should open wide the floodgates unless this would impact community trust. 
Throughout this phase of the project, the main focus was on construction of the Web site. The goal of the project was the creation of community, but the challenges of development took precedence. Until there was a site, there would not be a place where community could form. Asked "What is the ILF?" a majority of the research and development teams responded that it was the Web site, although many expressed the hope that it would become more-a community of teachers.

Generalized activity systems for the initial conception. At this point, 11 months into the project, we could begin the process of characterizing the ILF from an activity theory perspective. This generalized activity system was fairly straightforward, and our design and implementation process was not yet "complicated" with an appreciation of the STIN framework. Therefore, the generalized activity system was conceptualized based on reading through the data and then placing various labels within each of the components of Engeström's (1987) triangle. This illustration was revised until we had consensus among the core researchers that, indeed, the illustration captured the making of the ILF (see Figure 2).

In Figure 2, the participants (rsearchers and designers) are influencing the making of the ILF, which at this point was conceived as primarily an electronic structure. Various tools such as NSF funds, available technology, team-member expertise, related literature, online discussions, and connections supported the building of the e-ILF with other external experts (e.g., Apple Computer employees). The building of connections with the Indiana teaching community was supported by our team members who were former classroom teachers, by commitments from the Indiana Department of Education, and by our teacher advisory board. In addition to available tools, the rela-

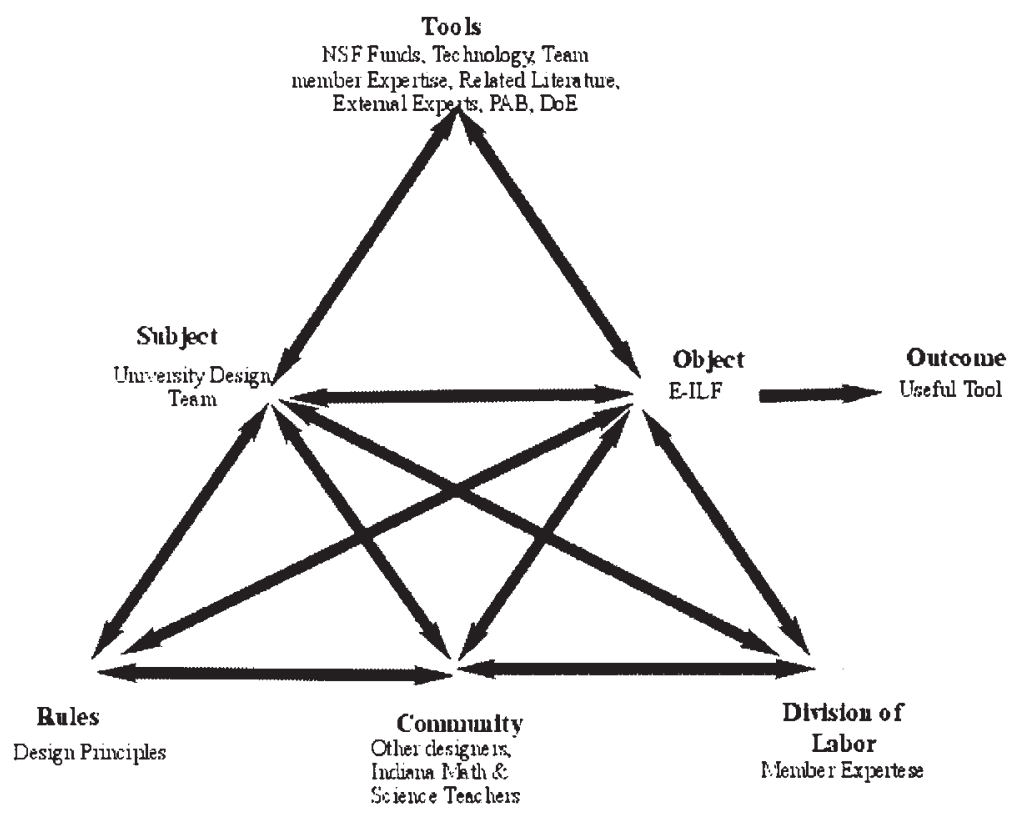

FIGURE 2 Generalized activity system of the making of the ILF conceived at inception of the project from a designer's perspective. 


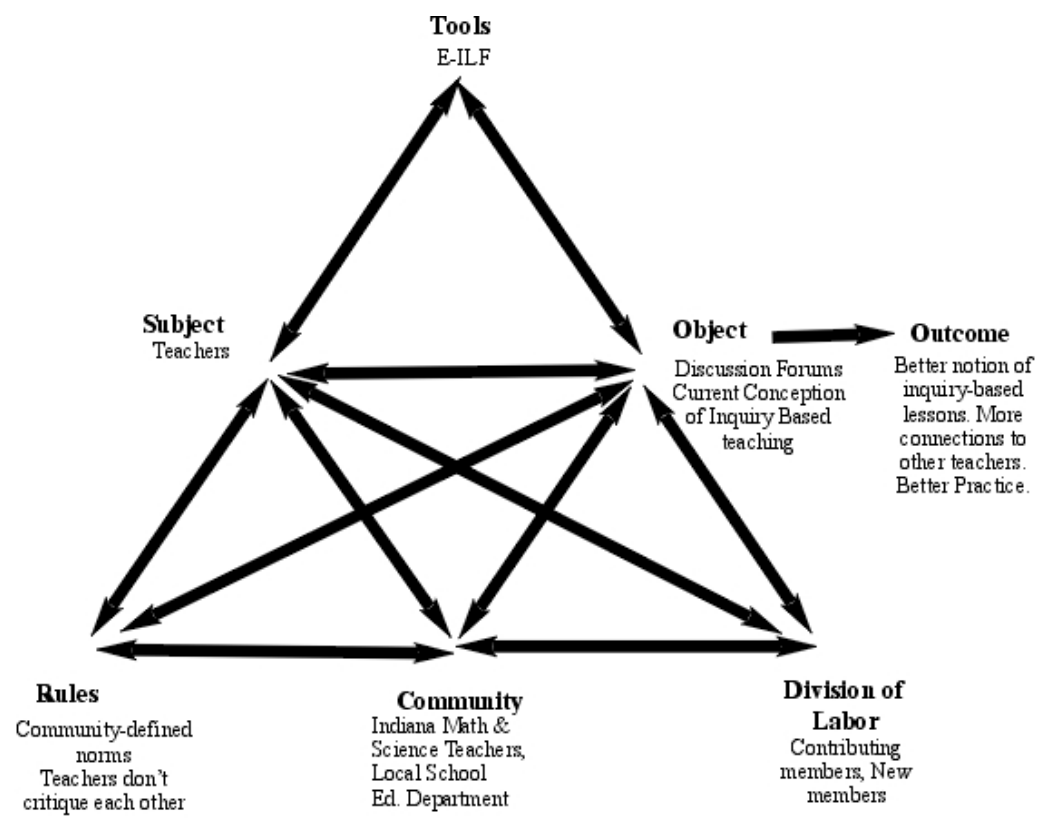

FIGURE 3 Generalized activity system of the use of the ILF conceived at inception of the project from a teacher's perspective.

tions of subject and object are also mediated by community, rules and norms, and division of labor. The communities include connections with the larger university research community, with Indiana math and science teachers, and even with the Apple technology community. In terms of division of labor, at this point in the development, the divisions simply involved the differences in design expertise among the project team members. Given the inexperience of our team at managing such a large project, many of the norms and rules were emergent, evolving in response to immediate project demands. Over time, however, we developed various technical (design) documents to manage some of the design process. The outcome at this point was primarily the usable e-ILF.

Whereas Figure 2 illustrates the generalized activity system for the making of the e-ILF, Figure 3 depicts how we initially envisioned the e-ILF to be used by teachers. Therefore, in Figure 3, the participants are the teachers. At this point the object of focus was their understanding of inquiry-based teaching, and the outcome was hypothesized to be better inquiry-based lessons in their classrooms and a more extensive network of connections to other teachers. The e-ILF primarily served as a tool to support these outcomes. Teachers were using the e-ILF in the context of the larger coalition of Indiana Math and Science teachers as well as in the context of their own school building (community). In terms of division of labor, our expectation was that there would be newcomers and more experienced "old-timers," with decisions based on some combination of teaching expertise and length of time as ILF members. Even though we had an idea of what we desired as appropriate rules and norms, we were willing to let the community develop these based on their participation and evolving needs. In addition, it was important to acknowledge that the current culture of teachers did not include publicly critiquing each other's classrooms. 


\section{Stage 2: From Usability to Sociability (12-24 Months From Project Inception)}

During the summer of 2000, as the 1st year of the project drew to a close, significant changes were under way. The Web site had been online for a couple of months, and six classrooms were available. Several hundred people had registered for the site. However, there was little activity in the discussion forums. Comments about tumbleweeds blowing through an empty town were common in design meetings. It was apparent that merely building it did not make teachers come.

Four significant events during this time fundamentally altered the project. First, in preparation for a conference presentation, several members of the research team began work on a paper that attempted to tell the story of the ILF from conception through implementation (this article is an outgrowth of that effort). Second, one of the PIs introduced the idea of STINs as a way to conceptualize the development of large projects. Third, the larger educational community began to publish more work related to building online communities so we could examine the research and design efforts of other groups. Working on this article and gaining understanding of the STIN of the ILF brought a much broader view of the ILF to the fore. Prior to this article, the ILF meant the Web site to most of the design team members. With the work on this article, it became common to refer to the Web site as the e-ILF, acknowledging the many other parts of the ILF. A design team member remarked later, "I was gone for a couple of weeks and when I came back, everyone was talking about the e-ILF and I wasn't sure if this was something new, or a new label, or what." Fourth, the conceptualization of the ILF as more than the e-ILF set the foundation for an expansion of outreach-through specialized subgroups, conference presentations, and in school workshops. This new view also changed the dynamic of the participation of research and development teams in the e-ILF.

During this time, meetings of the teacher advisory board and the research advisory board initiated significant change through feedback and reflection. These two groups suggested fundamental design changes, with the core commitment being that the ILF was far more than a Web site. In addition, during these meetings, and in light of conceptualizing the project as a STIN, the division among researchers, developers, and participants became much less distinct. Prior to this time, a tension existed between the facilitators supporting discussion by posting comments in the discussion boards and not wanting to muddy the waters or limit discussion by appearing to be experts giving the "final answer." However, when considered as part of a STIN, the ILF is everyone-teachers, researchers, development team, and advisory board members. Everyone is trying to create understanding and meaning. Everyone can and should post. With this new perspective, members of the development team, the participant advisory board, and interested teachers held posting parties where they met face to face, discussed issues raised on the discussion boards, and posted their considerations. Workshops to explore inquiry teaching and how the ILF could support teachers were held at Indiana University and at school sites. During the workshops, participants spent time reading and posting in discussion boards. The teacher liaison dedicated more time to supporting and encouraging participation. As more nonvirtual activities became part of the ILF, the name was changed to reflect the changing community. Originally called the Internet Learning Forum, the name was changed in October 2000 to the Inquiry Learning Forum, acknowledging both its online and face-to-face components. Community was becoming everyone who was associated with the ILF, both in person and online.

Significant questions raised by the teacher advisory board and the research advisory board centered on the usefulness of the Web site. "It's very nice, but why should I go there?" was a typical 
question. These considerations resulted in new features, with a recognition of the need to move from issues of usability to issues of sociability (Preece, 2000). Our major sociability design challenges were that of increasing connectedness and active participation. Our initial focus for increasing sociability was on design changes to the Web site. This included adding in a Workroom where teachers could go to collaboratively build inquiry-based lessons. Other methods for increasing participation included a new, more dynamic front page with featured classrooms (see Figure 4) and adding direct questions from videotaped teachers throughout their reflections on the lesson-thereby inviting comments from other community members.

Fostering networks of communities. During the first 6 months of the project, the concerns centered on "could it be built and how?" Now that the Web site was developed, a change in outlook occurred with the focus being much more on "how and why will it be used?" This change was the result of the reflections of the advisory boards as well as the reality that the e-ILF was up and running but had low levels of participation. The focus of the groups at Indiana University became how to encourage participation (primarily, this consisted of Web-site changes, some of which were previewed earlier).

As we entered the Spring 2001 semester, 9 months since launch and 15 months into the project, another change in our thinking of the ILF project occurred. Central to this change was that some of the more active teacher participants described themselves as part of a community, although much of the strength of this community was the face-to-face meetings. When the research and development teams met with these individuals, we no longer asked them to comment on "our" behavior but instead talked about what "we all" could do to encourage participation. It was here that we shifted from designers performing usability tests with our participants to all of us as collaborators with varying types of expertise and backgrounds. It was also here that we began to acknowledge that the ILF community was the participants, the tools, and the outcomes of our work.
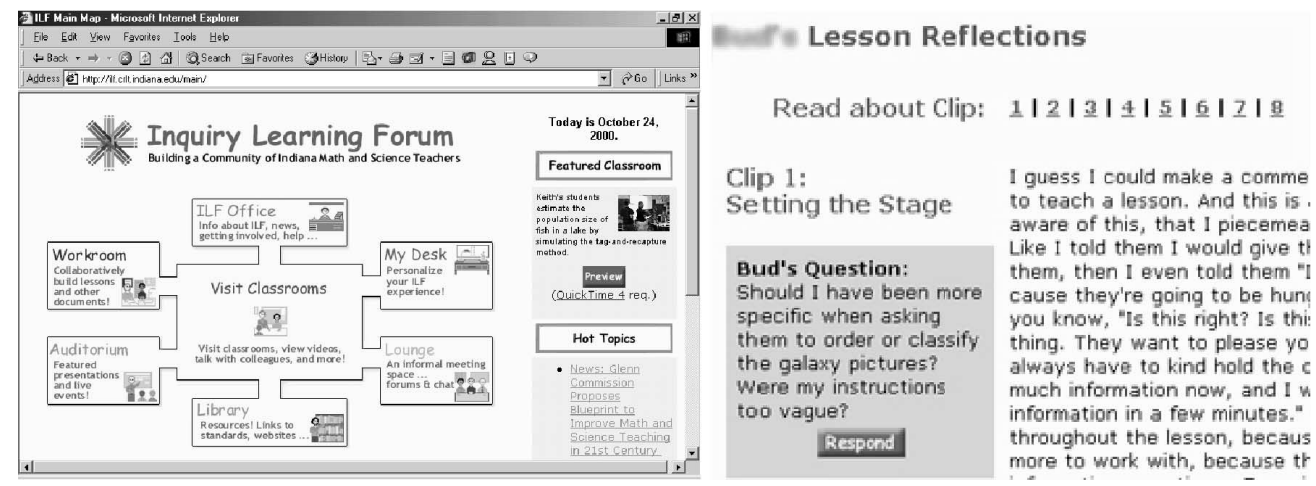

FIGURE 4 Current iteration of the ILF home screen, consisting of links to the Visit Classrooms, ILF Office, My Desk, Lounge, Library, Auditorium, and News. Also included is a current screenshot of a specific Classroom, including links to an overview of the lesson, reflective commentary, descriptions of teaching activity, lesson plans, student examples, and connections with both state and national standards. 
Second, there was an increased focus on more traditional professional development and outreach models - workshops, conference participation, and in-school presentations. Promoting and scheduling onsite presentations that introduced inquiry teaching and presented the e-ILF as a tool for ongoing development grew in importance as a community-building activity. We began to appreciate the importance of face-to-face connections as a precursor to online interactions. In fact, the notion of building online community has been severely challenged, and we now see ourselves as building a distributed network with online and face-to-face links.

In addition, although there had been some tension about letting preexisting groups en masse into the ILF because of fears that group members would ignore the broader community in favor of already established ties, a few semiprivate groups were allowed into the e-ILF. Different classes of preservice teachers were required to participate in special sections and invited their teachers to participate in the public sections. One of the co-PIs became PI of a grant of mathematics teachers building inquiry-based lessons. Those teachers used the e-ILF, particularly the new collaborative tool area (the Workspace), but also were welcome to participate in the general ILF community. In addition, the ILF was opened to allow registration on the e-ILF by administrators. Although the changes are still new, these efforts seem to be bearing fruit. There have been periods of increased communication, with a number of insightful and interesting posts from a variety of participants. "We are not yet at critical mass, but it feels like it's just around the corner," said the project manager. Further, during a recent meeting with a group of particularly active teachers, they expressed that they felt a part of an ILF community. We anticipate the final phase of the project may be termed integrated communities, where all the parts of the STIN of the ILF work together as communities of practice, informing each other. As stated earlier in a footnote, the ILF at the time of this revision (30 months into the project) had over 1,400 members who collectively accessed the Web site more than 20,000 times in December 2001.

\section{The ILF as a STIN}

We can now proceed to analyze our evolving conceptualization of the ILF as an activity system. By viewing the STIN of the ILF, the previous separate activity systems are no longer sufficient. The design and use activities are treated as transactional activities with each informing the other, challenging the practical value of acting and characterizing design and use activities as distinct, as is the case in Figures 2 and 3. These systems do not change so much as collapse into each other, with objects of separate systems becoming the parts of the larger object of this new system. When we initially conceptualized this new activity system, we had the object simply being the e-ILF or teachers' conceptions of inquiry. However, we felt uncomfortable with the object simply being the e-ILF because there were more things to act on than simply the e-ILF. The object was also troubling, for members acted not just on the Web site but on people in the ILF who participated in many ways - through workshops, face-to-face meetings, telephone, e-mail, and so forth. We concluded that, in addition to the STIN being the tool, the object of our conceived activity system was also the STIN of the ILF. This is because when a member uses the STIN as a whole or any part of the STIN, which includes critiquing an e-ILF classroom, dialoguing with another person in a copresent context, another person through the e-ILF as a tool, she is also transforming that same STIN (as an object and an outcome) by adding new information. Even as one person engages in ILF activity with the motive of altering his or her own practice, that personal growth feeds back and impacts the en- 
tire STIN. It was in part this realization that led us to conceptualize the STIN as defining the tools, the objects, the outcomes, and even the community. Indeed, the STIN permeates most of the system, taking on a different function when a tool than, for example, when an object. Although initially a conceptual challenge in that the process seems somewhat circular, this new transactional view of the activity system usefully informs the project. Later, in the implications, we discuss this process not as circular and contradictory but as complementary and therefore a useful extension of activity theory.

In our conceptualization of the ILF as STIN (see Figure 5), we can recognize actors that are not obvious to the usual consideration of Web sites but became apparent in conversations with teachers using the e-ILF. For instance, ILF members talked about influences on their choice of teaching practice such as students and parents concerned about potential SAT scores if didactic methods were not used to feed students the facts they needed to do well on standardized tests. Although we have very little ability to affect Educational Testing Services (producer of the SAT tests) and state standards, these comments indicated that ILF participants might want to educate parents and students about the benefits of inquiry teaching. Furthermore, this type of attention to parents and students and their needs for information has potential to change the nature of other transactions in the ILF in a dynamic way that is represented by the transactional STIN. A transaction that became very obvious in case studies of ILF members was the significance of school culture in affecting ILF participation. Put simply, teachers who had little support for their teaching methods on their

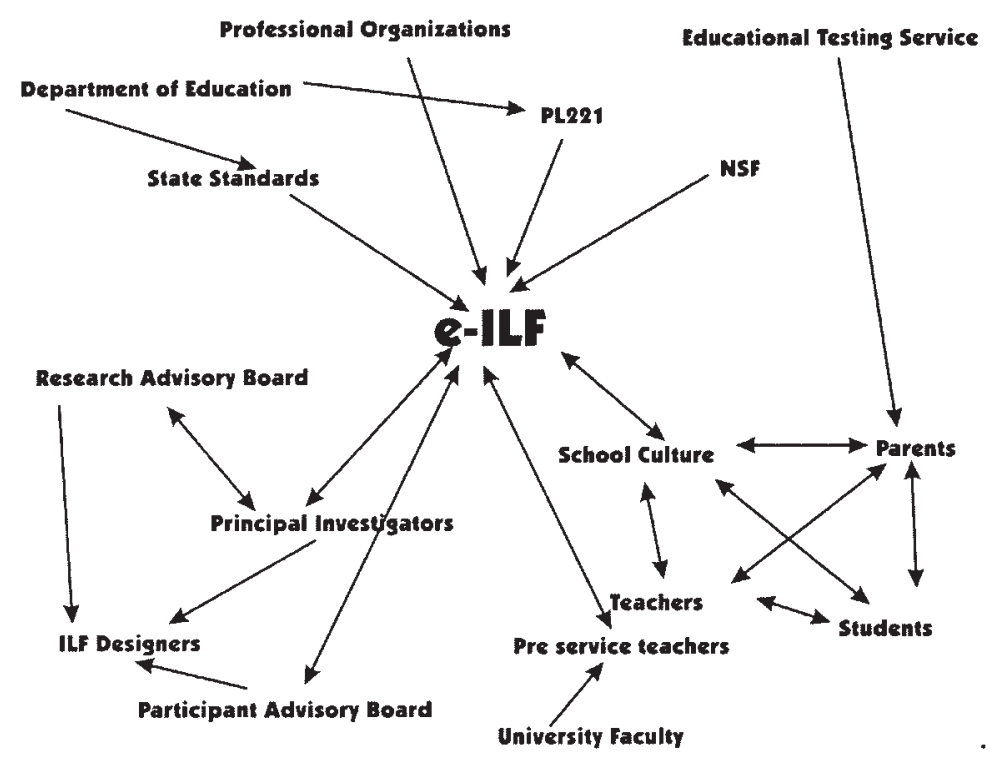

FIGURE 5 This figure shows our concept of the transactions and interactions of the e-ILF with other components of the STIN. It is important to note that not every potential transaction is displayed. We are only indicating, with arrows, the transactions and interactions we have observed in our fieldwork, interviews, and transcripts of online dialogue. Double arrows represent transactions where the transacting elements are changed in the process, whereas single-headed arrows indicate one-way quasi-causal interactions. 
school site were more likely to use the ILF than teachers who were satisfied with their in-school support. This speaks to the whole school reform that might be necessary when changing to inquiry pedagogies.

In the STIN of the ILF, illustrated in Figure 5, we have noted transactions (double-headed arrows) and actions (single-headed arrows) that we actually observed. There are many more potential connections among these actors and undoubtedly other possible actors (e.g., school administrators or politicians) that might be noticed as part of the STIN at another time. The connections we have drawn are the ones we have observed in our fieldwork, interviews with ILF members, and transcripts of online dialogue. It is important to differentiate this STIN from an "anything goes" system of transaction where every possible connection is noted. As we continue to do ethnographic research we expect to modify this diagram by adding new actors, removing some of the current actors, adding new transactions and interactions, and reviewing the currently represented ones.

Acknowledging the complexity of STINs does not mean that no boundaries exist and that everything can be grouped as one STIN. Rather, STINs have important boundaries and STINs transact; for example, the STIN of the ILF is alongside other communities that compete for the teachers' time. Or, for example, in addition to the outcome of member participation being the expansion of the STIN of the ILF, each teacher might also have an outcome in terms of his or her conception of inquiry-based learning or ideas for a lesson the following day. Researchers might also have an anticipated outcome of developing new research in addition to their design focus of improving this particular STIN (Brown, 1992). These other factors may compete against the goals of the ILF, creating fundamental tensions that influence the potential of the STIN to grow. The balancing of system tensions (e.g., university researcher vs. schoolteacher, ILF community vs. local school community, getting lesson plans vs. transforming notions of inquiry-based learning) is what allows the STIN of the ILF to grow and prosper or stagnate and disappear.

In this new conception, the STIN is not one part of the activity system but, instead, permeates the entire activity system, with the system components simply referring to different functions of the STIN. This is in part because, in a community model of learning, the community members are working on themselves, and through their transactions, they transform themselves and the network that is the STIN of which they are a part. However, just as we can collapse the activity theory triangles represented in Figures 2 and 3 into a STIN framework (represented in Figure 5), we can employ activity theory as a useful analytical lens for making sense and characterizing the STIN. This notion positions the function of Engeström's triangle as a theory for understanding functional significance and not as a theory about ontological divisions, ${ }^{3}$ thereby minimizing potential dualities and highlighting transactions both at the inter- and intracomponent level (Garrison, 2001). In the new activity system conception, the subject is the ILF community, including members as well as designers and researchers. Although the e-ILF is certainly the primary tool being used by participants, the e-ILF is now viewed as part of the larger collective (the STIN), all of which constituted the tool of the ILF. It is also the object, as all parts of the network transact, recreating themselves. The resultant outcome is again the STIN of the ILF, with the STIN also taking

\footnotetext{
${ }^{3}$ This discussion is consistent with Garrison's (2001) critique of activity theory as being interactional and not transactional. We return to this point in the Implications section, but the reader is also directed toward Garrison's article for a more complete discussion of Dewey's distinction between interaction and transaction and how the notion of transaction could enrich activity theory.
} 
on the function of object, community, and tool at different points in time and from the perspective of different members.

The rules are a result of the transactions within the STIN and include gatekeeping activities, time and resource constraints, and the ways in which teachers critique each other. As opposed to top-down rules, predefined for the community, the rules and norms emerge from the community. However, because teachers are not used to critique each others' teaching (i.e., it is not part of teacher norms even in face-to-face contexts), we are exploring active interventions that can stimulate critical dialogue. Although university researchers may advocate inquiry as the goal for teaching, teachers who have multiple constraints in terms of class size, time, meeting standards, and so forth, may not see inquiry-based teaching as the most useful approach. Finally, the division of labor moves to a "we all create, we all learn" model, with an explicit attempt to minimize the division among university researchers and schoolteachers. The goal is to have everyone be designers and users of the system. However, there is an internal tension with respect to division of labor: Designers have the most power (they have more time to spend on development - it is, after all, their job), contributing members who put up videos rank second in terms of power, and last there are the everyday participants who have limited access to defining the community.

The temptation is to look at any STIN or activity system as a black box, static in both time and structure. However, it is important to note that this is only a macroreflection. The generalized account of the ILF activity system (or of the STIN of the ILF) obfuscates the numerous nested levels of activity that occur throughout the making of the ILF. Thus, any model of the activity system is not a static model, but a model continually in the making (Latour, 1987). Every system, including the ILF, has a history and nested actions, which when viewed from different vantage points and from different points in time may be construed and represented differently and constitute their own activity systems.

\section{CONCLUSIONS}

Earlier, we told the story of the design and implementation of the ILF. The story began with a number of dichotomies that guided our thinking and work. In our initial work, there was a division between the design activity and the use activity. The former activity system was very much determined by the research and design team, with the users of the system simply being participants who, through needs analysis and usability tests, would have an influence on the object of focus-the e-ILF. The outcome of our design work was expected to be an electronic structure that would become a tool for teachers through which they would develop a deeper understanding of inquiry-based teaching and become a member of a community. Over time, however, we realized that building community implies much more than having a good design. In fact, notions of usability become less useful than notions of sociability, switching the emphasis from human-computer interactions to human-human interactions, human-human interactions as mediated by technology, and human-computer transactions.

Similarly, an important shift in thinking over the course of the project was from viewing the ILF as an electronic forum to viewing the ILF as a STIN (Barab et al., 2001; Kling et al., 2001). This shift began with an appreciation that teachers were not simply critiquing videos or conversing with colleagues but were critiquing other teachers in a permanent, semipublic forum. Looking at the online classes as intimate public portrayals of individual teachers as opposed to technical ar- 
tifacts suggested that the exhibited teachers needed to publicly express their desire for feedback and that we had to work more intimately with teachers in modeling feedback and supporting them in critiquing other teachers in useful ways. Appreciation of the ILF as an STIN also pushed us to more readily acknowledge the social component and, as a result, support more face-to-face interactions among members. We began hosting face-to-face workshops, sending direct e-mails to teacher participants, and spending more time in schools connecting with teachers. Our notion of the ILF transitioned from an online community to a Web-based or Web-supported community. Finally, this meant supporting preexisting and bounded groups working together in the ILF.

At the project's inception, our focus was much more on creating a place where individual teachers across the state would come and find community. We now have small groups that have already formed and that can come to form their own bounded communities, meeting face-to-face and then continuing work within the e-ILF. Such groups need intimate working areas where members can collaborate on a focused task (developing a lesson) or a mutual enterprise (investigating water quality using a common curricular framework) and evolve their notions of inquiry. Given the targeted focus, these groups might complete their work and leave the ILF, not participating in the growth of a broader network of support. A clear challenge is to determine how to balance the opportunities, providing teachers with particular tasks and with an intimate group while stimulating their interest and engagement in the community at large (Berg, 1999).

In addition to sociability concerns of the interface itself (Preece, 2000), the building of community is not something that can necessarily be done from the outside. Communities of practice are organic systems that emerge through interactions among community members, not because some designer made it so (Barab et al., 1999; Wenger, 1998). Therefore, a major design shift occurred when we began collapsing the us-them (designers vs. teacher community members) distinction. In addition to changes in the design team's (expanded to include all ILF members) everyday practices, this resulted in a change in how we characterized ILF activity. Initially, we characterized ILF activity in terms of two systems (one for designers, Figure 2, and another for ILF users, Figure 3 ), but in Figure 5 we characterized current ILF activity in terms of one system that integrates all ILF users and minimizes the distinction between the designers and the users of the system-as well as acknowledges the transactional functioning of the system as a STIN.

Finally, we have argued that no single theory or concept is sufficient for telling the complete story in building successful online communities. Although activity theory clearly helped us illuminate some of the tensions in our own work (see Figures 2 and 3), we feel that understanding the messiness of designing complex systems such as online communities is best underscored through the use of multiple models of analysis that complement each other. This is in no small part because of the transactional nature of these systems, thus challenging the ontological compartmentalization of Engeström's (1987) scheme into components such as the tool, the subject, the object, and the outcome. Instead, we viewed the ILF as operating as a STIN that occupied most components of the activity system, whereas the function of the STIN changed in terms of whether we were looking at the STIN through the lens of subject, tool, object, or outcome. It is through an examination of these functions that conceptualizing the designing of an online community both in terms of a STIN and in terms of an activity system becomes complimentary and useful.

By examining how the STIN operated as a tool, as an object, as an outcome, or as a community, we were able to gain more information with respect to the effectiveness of the STIN more generally and to develop useful design interventions. Clearly, though, the important point was not to 
treat these components as isolated but instead to examine the STIN within each component in terms of the overall transactional dynamics of the system. In this way, our treatment of the ILF as a STIN and the STIN of the ILF as an activity system offered insights into the interrelations of people and technology, participation and reification, identity and online place, designed and emergent, and local constraints and global reform. It is in this way that the two characterizations are more than complementary perspectives. They are synergistic in that together the two theoretical approaches do more than either one individually, as well as more than if we added together their unique contributions.

Activity theory and STIN, taken in combination, provide a richer view of the development and actualization of a system than either can on its own. For example, although activity theory offers an excellent reification of the dynamics of a system, it also encourages the ontological compartmentalization of reciprocally defining and transacting components. The STIN view encourages the consideration of the multitude of interdependent mutually transacting players but tends to account for everything and therefore has less utility as an explanatory lens. We found it useful to conceptualize the ILF as a STIN and then use an activity theory framework to focus our analysis on particular functions of the STIN. Taken together, activity theory and STIN frameworks provide harmonizing perspectives for understanding the components of activity as well as their interrelations.

\section{IMPLICATIONS}

In this article, we applied activity theory as an analytical lens for characterizing the process of designing and supporting the implementation of a community of practice. Specifically, we built on Engeström's characterization of activity systems, using his model as a way of conceptualizing the various design and use activities. In addition, we adopted the notion of STIN as a second theoretical lens that, when coupled with activity theory, provides important insights into the design of online community and the characterization of community functioning.

Our findings point toward three implications. First, activity theory can provide a useful analytical tool for characterizing design activity, especially in terms of illuminating the challenges of designing something like community. The model proved useful for understanding our design process, supporting insights in terms of the challenges and decision-making process. Its utility was first apparent when we attempted to characterize the system activity, resulting in two different systems - one for the users and one for the designers. As we attempted to determine how to relate these two systems, we realized the schism in our design work. Although the team was already becoming uncomfortable with the divide, characterizing activity in terms of two distinct systems made this even more apparent. The notion of looking at design activity not as simply the development of a tool or object but as developing a system to support activity helps the designer gain richer insights into the use process. Looking beyond the subject-object as mediated by tool interaction to the larger community (STIN) in which mediation is viewed as transactional and symmetrical also proved useful in that it helped us better understand the clashes of the system we were developing and the culture and community of use in which our users were enculturated and to which they were accountable.

Second, as we moved toward trying to design a community, especially one in which the members are expected to engage in new practices that challenge their current culture, many tensions 
emerged. At one level, it is presumptuous of one culture to assume they know what is best for another culture. Who are we as university researchers in our ivory towers with our relaxed teaching schedules to tell classroom teachers who sometimes meet for 30- to 45-min class blocks daily and who have national standards to which their students are held accountable that they need to transform their teaching practices? Even if we were justified in deciding what constitutes "best practices" for teachers, do we also have the ability to decide and build their community? More generally, can anything resembling "community" be designed?

We argue that designing community is a complex activity that must allow for evolving, not preordained, development. The former acknowledges community as a bubbling, emergent system that evolves from the inside, the direction of which is a natural outgrowth and is defined through the interactions of its members. Barab, Barnett, and Squire (2002) argued that

a central challenge for educators who are designing and facilitating communities of practice in the service of learning is to recognize the system tensions, identify how they impact community life, balance their influence, minimize potentially damaging conflicts, and allow the system to evolve as the community as a whole learns to balance the multiple needs of its members. (p. 32)

Although community facilitators can use broad guidelines of facilitation and community building to foster communities for learning (Kim, 2000), the process by which members negotiate meaning within a community is a complex one that draws on rich sets of dynamics and local tensions.

Since Lave and Wenger's (1991) seminal book on communities of practice, it has become generally accepted to look at community in which action is situated as an essential mediating artifact of action. This is particularly true when viewing communities of practice designed to support learning (Barab, Kling, \& Gray, in press), where the community itself is a tool that mediates the interaction between the subject and object. In terms of Engeström's triangle, this treatment elevates the notion of community from simply occupying the bottom of the triangle to an entity whose reach is distributed across multiple components as it functions as tool; object; outcome; and, at one unit of analysis, even subject. As such, treating community as a learning intervention—a tool—compartmentalizes experience into separate components. Instead, when the community itself is considered a tool, it comes to occupy multiple components, with its compartmentalization being an acknowledgment of function - not form. Even when perceived through its various functions, the community does not interact with itself, but instead the community in its multiple roles continually transacts so that each action changes the nature of the community as a whole.

The third and final implication is that the conceptualization of an activity system as discussed by Engeström benefits from the examination of STINs of evolving communities of practice over time. Although each of the existent points of an activity triangle representation remains in place, each point is a lens into the whole, dynamically evolving STIN. Examination of the tensions between the points of view may provide excellent information, but in such systems it is crucial to recognize that each point is not a part of the whole but is merely a different view of a system that continually remakes itself based on internal activity. Over time, as the ILF creates itself, the activity system of the STIN of the ILF turns inward, with each lens of the Engeström triangle informing and creating the others. It is in this way that activity systems can be described as transacting, not simply interacting (Garrison, 2001).

Whereas Engeström's triangle provides an analytical focus and allows researchers to gain insight into the interaction among the components of the triangle, it also compartmentalizes them 
and as such treats them as independent ontological entities, essences, or realities. Transactional thinking, however, "allows us to see things as belonging together functionally ... [and] allows us to recognize them as subfunctions of a larger function [the ILF]" (Garrison, 2001, p. 23). By functionally relating each component as subfunctions of the ILF system, we come to appreciate how the ILF functions as a unity and the ways in which it comes to constitute multiple components of an activity. Therefore, although an activity theory framework as conceptualized by Engeström $(1987,1993)$ was useful for understanding this process and some of our faulty design decisions, isolating components to particular locations along the triangle did not appear to be ontologically consistent with the activities through which this community of practice was made. It is in this way that we came to appreciate the value of using a STIN framework for capturing the complex and transactional nature of the system and the value of activity theory for helping us understand the multiple functions of the STIN. Therefore, both worked at levels of analysis that also allowed us to do valuable theoretical, analytical, and practical work.

In closing, what began as an examination of a tool using activity theory as a lens has resulted in the lens being turned back on itself. As activity theory informed the dynamic activity of the creation of a STIN, so the STIN informed the dynamic nature of activity theory. When tools are not ready-made artifacts (a toaster, Word 6.0, or even a manuscript) but constitute continually evolving systems, each system is defined by and defines the participants who participate in its use and continual remaking. Because our tool (the ILF community) is part of a reform effort, the tool is also being continually reformed through its use and remaking. The community and its members, conceived as a STIN, were the subject, tool, object, and even the outcome, challenging the ontological validity of treating components of the activity system as mutually exclusive. And so, viewing the STIN through the lens of tool, subject, and so forth, and viewing how the STIN interacts in these different roles provides a rich understanding of the STIN. Viewing the parts of the activity system as parts of the STIN provides a richer understanding of the activity system. Taken together, these two analytical lenses provide a micro- and macroview of the ILF and, more generally, the design of community. We believe that it is through the application of complementary theoretical perspectives, especially when their assumptions employ us to acknowledge multiple scales and foci for analyses, that theory can have the greatest practical significance.

\section{ACKNOWLEDGMENTS}

This research was supported in part by a Knowledge and Distributed Intelligence Grant from the National Science Foundation, REC-9980081. A version of this manuscript was presented at the American Educational Research Association in Seattle, Washington, in April 2001. Special thanks to members of our design team and to all the teachers who have spent hours developing our system. Also, special thanks to Scott McDonald, Don Cunningham, Julie Moore, and the anonymous MCA reviewers for their valuable feedback. Thanks to Rob Kling for his insights and for the numerous discussions, which provided the intellectual foundation for this manuscript.

\section{REFERENCES}

Barab, S. A., Barnett, M. G., \& Squire, K. (2002). Developing an empirical account of a community of practice: Characterizing the essential tensions. The Journal of the Learning Sciences, 11, 489-543. 
Barab, S. A., Barnett, M., Yamagata-Lynch, L., Squire, K., \& Keating, T. (2002). Using activity theory to understand the systemic tensions characterizing a technology-rich introductory astronomy course. Mind, Culture, and Activity, 9, 76-108.

Barab, S. A., Cherkes-Julkowski, M., Swenson, R., Garrett. S., Shaw, R. E., \& Young, M. (1999). Principles of self-organization: Ecologizing the learner-facilitator system. The Journal of the Learning Sciences, 8, 349-390.

Barab, S. A., \& Duffy, T. (2000). From practice fields to communities of practice. In D. Jonassen \& S. M. Land (Eds.), Theoretical foundations of learning environments (pp. 25-56). Mahwah, NJ: Lawrence Erlbaum Associates, Inc.

Barab, S. A., Kling, R., \& Gray, J. (Eds.). (in press). Designing for virtual communities in the service of learning. Cambridge, MA: Cambridge University Press.

Barab, S., MaKinster, J. G., Moore, J., Cunningham, D., \& the ILF Design Team. (2001). Designing and building an online community: The struggle to support sociability in the Inquiry Learning Forum. Educational Technology Research and Development, 49(4), 71-96.

Barab, S. A., MaKinster, J., \& Scheckler, R. (2003). Designing system dualities: Characterizing a web-supported teacher professional development community. Information Society, 19, 237-256.

Brown, A. L. (1992). Design experiments: Theoretical and methodological challenges in creating complex interventions in classroom settings. The Journal of the Learning Sciences, 2, 141-178.

Dewey, J., \& Bentley, A. (1989). Knowing and the known. In J. A. Boydston (Ed.), John Dewey: The later works, Volume 16 (pp. 1-279). Carbondale: Southern Illinois University Press. (Original work published 1949)

Engeström, Y. (1987). Learning by expanding. Helsinki, Finland: Orienta-konsultit.

Engeström, Y. (1993). Developmental studies of work as a testbench of activity theory: The case of primary care medical practice. In S. Chaiklin \& J. Lave (Eds.), Understanding practice: Perspectives on activity and context (pp. 64-103). Cambridge, UK: Cambridge University Press.

Engeström, Y. (1994). Teachers as collaborative thinkers: Activity-theoretical study of an innovative teacher team. In I. Carlgren, G. Handal, \& S. Vaage (Eds.), Teachers minds and actions: Research on teachers' thinking and practice. London: Falmer.

Engeström, Y. (1999). Activity theory and individual and social transformation. In Y. Engeström, R. Miettinin, \& R. Punamaki (Eds.), Perspectives on activity theory (pp. 19-38). New York: Cambridge University Press.

Garrison, J. (2001). An introduction to Dewey's theory of functional "trans-action": An alternative paradigm for activity theory. Mind, Culture, and Activity, 8, 275-296.

Guba, E. G., \& Lincoln, Y. S. (1983). Epistemological and methodological bases of naturalistic inquiry. In G. F. Madaus, M. S. Scriven., \& D. L. Stufflebeam (Eds.), Evaluation models: Viewpoints on educational and human services evaluation (pp. 311-334). Boston: Kluwer-Nijhoff.

Jonassen, D. H., \& Rohrer-Murphy, L. (1999). Activity theory as a framework for designing constructivist learning environments. Educational Technology Research and Development, 47(1), 61-79.

Kim, A. J. (2000). Community building: Secret strategies for successful online communities on the Web. Berkeley, CA: Peachpit Press.

Kling, R., \& Courtright, C. (in press).Characterizing collective behavior online: The social organization of hangouts, clubs, associations, teams and communities. In S. Barab, R. Kling, \& J. Gray (Eds.), Designing for virtual communities in the service of learning. Cambridge, UK: Cambridge University Press.

Kling, R., McKim, G., Fortuna, J., \& King, A. (2001). A bit more to IT: Scientific communication forums as socio-technical interaction networks. Unpublished manuscript.

Kuutti, K. (1996). Activity theory as a potential framework for human-computer interaction research. In B. Nardi (Ed.), Context and consciousness: Activity theory and human-computer interaction (pp. 17-44). Cambridge, MA: MIT Press.

Latour, B. (1987). Science in action: How to follow scientists and engineers through society. Milton Keynes, England: Open University Press.

Lave, J., \& Wenger, E. (1991). Situated learning: Legitimate peripheral participation. New York: Cambridge University Press.

Leont'ev, A. (1974). The problem of activity in psychology. Soviet Psychology, 13(2), 4-33.

Leont'ev, A. (1981). Problems of the development of mind. Moscow: Progress.

Leont'ev, A. (1989). The problem of activity in the history of Soviet psychology. Soviet Psychology, 27(1), 22-39.

Lincoln, Y. S., \& Guba, E. G. (1985). Naturalistic inquiry. Beverly Hills, CA: Sage.

Miettinen, R, (1997). The concept of activity in the analysis of heterogeneous networks in innovation process. CSIT Workshop, “Actor Network and After." Retrieved January 20, 2002, from http://communication.ucsd.edu/MCA/Paper/Reijo/Reijo.html 
Nardi, B. (Ed.). (1996). Context and consciousness: Activity theory and human-computer interaction. Cambridge, MA: MIT Press.

Preece, J. (2000). Online communities: Designing usability, supporting sociability. Chichester, UK: Wiley.

Schuler, D. \& Namioka, A. (Eds.). (1993). Participatory design: Principles and practices. Hillsdale, NJ: Lawrence Erlbaum Associates, Inc.

Scriven, M. S. (1983). Evaluation methodologies. In G. F. Madaus, M. S. Scriven, \& D. L. Stufflebeam (Eds.), Evaluation models: Viewpoints on educational and human services evaluation (pp. 229-260). Boston: Kluwer-Nijhoff.

Smith, M. A., \& Kollock, P. (Eds.). (1999). Communities in cyberspace. London: Routledge.

Wenger, E. (1998). Communities of practice: Learning, meaning, and identity. Cambridge, UK: Cambridge University Press. 